\title{
METNet: A combined missing transverse momentum working point using a neural network with the ATLAS detector
}

\author{
Benjamin Hodkinson*, on behalf of the ATLAS Collaboration \\ University of Cambridge \\ E-mail: ben.hodkinson@cern.ch
}

In order to suppress pile-up effects and improve resolution, the ATLAS experiment at the LHC employs a suite of working points for missing transverse momentum $\left(p_{\mathrm{T}}^{\text {miss }}\right)$ reconstruction, and each is optimal for different event topologies and different beam conditions. A neural network (NN) can exploit various event properties to pick the optimal working point on an event-by-event basis, and also combine complementary information from each of the working points. The resulting regressed $p_{\mathrm{T}}^{\text {miss }}$ (METNet) offers improved resolution and pile-up resistance across a number of different topologies compared to the current $p_{\mathrm{T}}^{\text {miss }}$ working points. Additionally, by using the NN's confidence in its predictions, a machine learning-based $p_{\mathrm{T}}^{\text {miss }}$ significance ('METNetSig') can be defined. This contribution presents simulation-based studies of the behaviour and performance of METNet and METNetSig for several topologies compared to current ATLAS $p_{\mathrm{T}}^{\text {miss }}$ reconstruction methods.

\footnotetext{
*** The European Physical Society Conference on High Energy Physics (EPS-HEP2021), ***

*** 26-30 July $2021 * * *$

*** Online conference, jointly organized by Universität Hamburg and the research center DESY ***
}

\footnotetext{
*Speaker.
} 


\section{Introduction}

Missing transverse momentum $\left(p_{\mathrm{T}}^{\text {miss }}\right)[1]$ is defined as the magnitude of the negative vectorial sum of the transverse momentum of all the visible objects produced in the hard scatter of a protonproton collision event in the ATLAS detector [2] at the LHC. ATLAS employs several working points for $p_{\mathrm{T}}^{\text {miss }}$ reconstruction, each of which is optimal for different event topologies and levels of pile-up. The working points considered here are: Loose, Tight, Tighter and Tenacious. These have increasingly strict selections on hadronic jets. The variable $p_{\mathrm{T}}^{\text {miss }}$ significance [3] is also used to separate processes with 'real' $p_{\mathrm{T}}^{\text {miss }}$ (from genuine invisible particles, such as neutrinos) and 'fake' $p_{\mathrm{T}}^{\text {miss }}$ (from detector mis-measurement and pile-up).

This contribution presents the methods and results of training a neural network (NN) to pick and combine the $p_{\mathrm{T}}^{\text {miss }}$ working points for each event to produce a new $p_{\mathrm{T}}^{\text {miss }}$ definition ('METNet'), and also to define a machine learning-based $p_{\mathrm{T}}^{\mathrm{miss}}$ significance ('METNetSig'). Further details on this study can be found in Ref. [4].

\section{Neural network architecture}

The NN is a multi-layer perceptron trained on about three million $t \bar{t}$ and di-boson MC events. The NN predicts generator-level $p_{x, y}^{\text {miss }}\left(p_{x, y}^{\text {miss, True }}\right)$ given 60 input features including $p_{\mathrm{x}, \mathrm{y}, \mathrm{T}}^{\text {miss }}$ predictions for the Loose, Tight, Tighter and Tenacious working points, plus additional information characterising pile-up and event topology, such as number of primary vertices and mean number of interactions per bunch crossing.

Results are shown for a NN trained using two loss functions:

1. $\mathcal{L}=\mathcal{L}_{\text {Huber }}= \begin{cases}\frac{1}{2}(y-\hat{y})^{2} & ,|y-\hat{y}| \leq \delta \\ \delta|y-\hat{y}|-\frac{1}{2} \delta^{2} & , \text { otherwise }\end{cases}$

2. $\mathcal{L}=\mathcal{L}_{\text {Huber }}+\mathcal{L}_{\text {Sinkhorn }}$

where $y$ and $\hat{y}$ are the NN's prediction and target respectively and $\delta$ is set to $\frac{3}{2}$. Here $\mathcal{L}_{\text {Sinkhorn }}$ is the Sinkhorn distance between the output and target batch which is included to reduce a bias in the distribution of the NN's predictions. Sample weights are also used to reduce a bias towards the bulk of the training set. The weight of each event is calculated using the reciprocal of the $p_{\mathrm{T}}^{\text {miss, True }}$ histogram of the training set.

The training and testing sets are passed through two pre-processing steps. First, to remove $\phi$ invariance from the inputs each event is rotated such that $p_{\mathrm{T}}^{\text {miss, Tight }}$ points along the $x$-axis. Then, each input/output feature is standardised by subtracting the mean and dividing by the standard deviation. The NN outputs are transformed by the inverse of these steps.

\section{METNet: Resolution and bias}

Figure 1 shows the root-mean square of the deviation from truth of the $p_{x, y}^{\text {miss }}$ predictions (ie. the resolution) for the four working points and METNet both with and without the Sinkhorn loss. METNet has improved resolution compared to the $p_{\mathrm{T}}^{\text {miss }}$ working points for both topologies. The performance for $Z \rightarrow \mu \mu$ events and stability against pile-up is particularly notable, as this is a topology which was not seen by the $\mathrm{NN}$ during training and which contains no real $p_{\mathrm{T}}^{\text {miss }}$. 


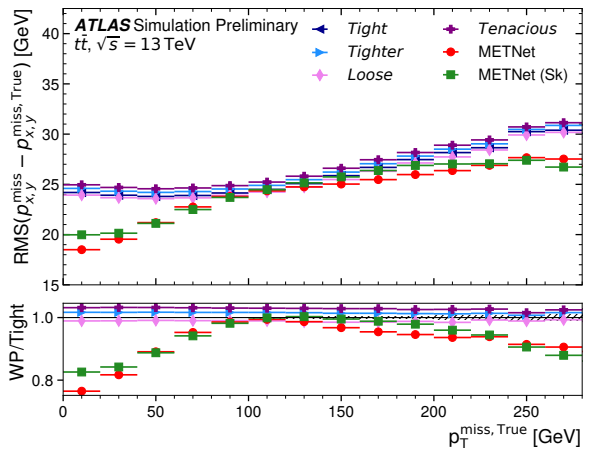

(a) $t \bar{t}$

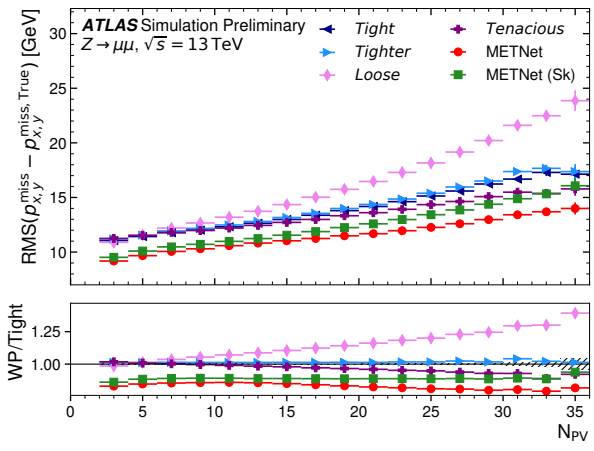

(b) $Z \rightarrow \mu \mu$

Figure 1: Root-mean-square of the difference between the predicted value of $p_{x, y}^{\text {miss }}$ and $p_{x, y}^{\text {miss, True }}$ for (a) $t \bar{t}$ events in bins of $p_{\mathrm{T}}^{\text {miss, True }}$, (b) $Z \rightarrow \mu \mu$ events in bins of number of primary vertices. METNet is shown with and without the Sinkhorn (Sk) loss, along with current $p_{\mathrm{T}}^{\text {miss }}$ working points. The lower panel shows the ratio with respect to the Tight working point, and the hatched band indicates the statistical uncertainty for the Tight working point [4].



Figure 2: $p_{\mathrm{T}}^{\mathrm{miss}}$ distribution for METNet (with and without the Sinkhorn loss), $p_{\mathrm{T}}^{\text {miss, True }}$ and four $p_{\mathrm{T}}^{\text {miss }}$ working points, for $t \bar{t}$ events. Events are unweighted [4].

Figure 2 shows the $p_{\mathrm{T}}^{\mathrm{miss}}$ distribution for METNet (both with and without the Sinkhorn loss). Including the Sinkhorn contribution to the loss improves the agreement of the METNet distribution with $p_{\mathrm{T}}^{\text {miss, True }}$ for $0<p_{\mathrm{T}}^{\text {miss }}<100 \mathrm{GeV}$.

\section{METNetSig}

The $p_{\mathrm{T}}^{\text {miss }}$ significance is defined by weighting a $p_{\mathrm{T}}^{\text {miss }}$ prediction by a 'confidence', $\sigma$. The state-of-the-art ATLAS implementation is an object-based $p_{\mathrm{T}}^{\text {miss }}$ significance [3], for which $\sigma$ is a function of the $p_{\mathrm{T}}$-dependent resolutions of the objects which enter the $p_{\mathrm{T}}^{\text {miss }}$ calculation. To define a machine-learning based $p_{\mathrm{T}}^{\text {miss }}$ significance, the Huber loss function is replaced with the 
Gaussian negative log likelihood (GNLL) loss, $\mathcal{L}_{\mathrm{GNLL}}=\log \sigma_{x, y}+0.5\left(\frac{p_{x, y}^{\text {miss, NN }}-p_{x, y}^{\text {miss, True }}}{\sigma_{x, y}}\right)^{2}$. The resulting NN has four outputs, $\left(p_{x}^{\text {miss, NN }}, \sigma_{x}, p_{y}^{\text {miss, NN }}, \sigma_{y}\right.$ ), from which METNetSig is defined as METNetSig $=p_{\mathrm{T}}^{\text {miss, NN }} / \sigma$.

Figure 3 shows (a) METNetSig and (b) object-based $p_{\mathrm{T}}^{\text {miss }}$ significance [3] for a supersymmetric signal process plus two Standard Model backgrounds. METNetSig has a similar shape to objectbased $p_{\mathrm{T}}^{\text {miss }}$ significance for each topology, but with a lower cut-off point. Note that the NN learns $\sigma$ independently of the object-based measurements that are inputs to object-based $p_{\mathrm{T}}^{\text {miss }}$ significance. Nonetheless, METNetSig has similar real $p_{\mathrm{T}}^{\text {miss }}$ vs fake $p_{\mathrm{T}}^{\text {miss }}$ separation power.

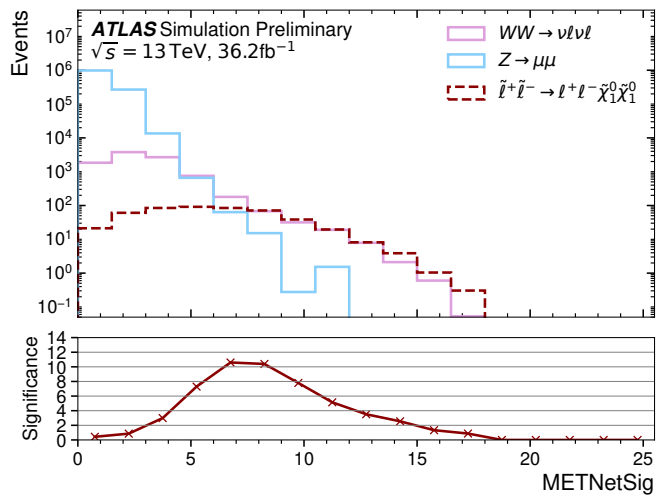

(a)

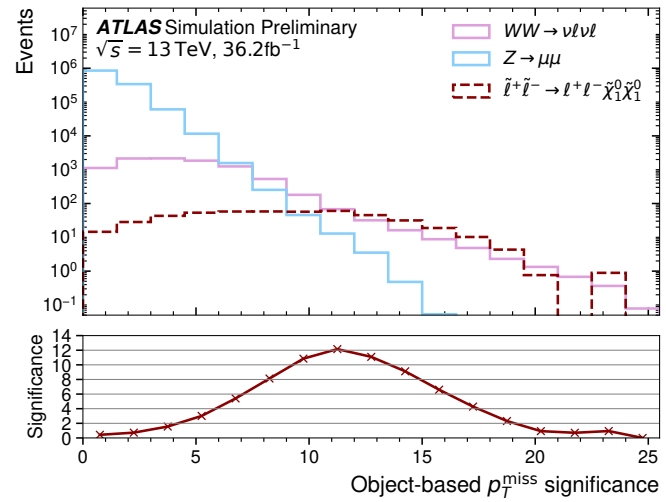

(b)

Figure 3: Distributions of (a) METNetSig and (b) object-based $p_{\mathrm{T}}^{\text {miss }}$ significance for a supersymmetric signal point and $W W$ and $Z \rightarrow \mu \mu$ backgrounds. The lower plot shows the signal significance for a lower-bound cut at each $x$-axis bin value [4].

\section{Conclusion}

This contribution presents the performance of METNet and METNetSig - variables defined using the outputs of a neural network - compared to current ATLAS methods. METNet has significantly improved resolution for a range of topologies, including those not seen during training. Including a Sinkhorn contribution to the loss function reduces an observed negative bias in the NN's predictions. METNetSig shows similar behaviour per-topology to object-based $p_{\mathrm{T}}^{\text {miss }}$ significance, and can distinguish between real $p_{\mathrm{T}}^{\text {miss }}$ and fake $p_{\mathrm{T}}^{\text {miss }}$.

\section{References}

[1] ATLAS Collaboration, Eur. Phys. J. C 78 (2018) 903 [1802 . 08168].

[2] ATLAS Collaboration, JINST 3 (2008) S08003.

[3] ATLAS Collaboration, Tech. Rep. ATLAS-CONF-2018-038, CERN, Geneva (Jul, 2018).

[4] ATLAS Collaboration, Tech. Rep. ATL-PHYS-PUB-2021-025, CERN, Geneva (Jul, 2021). 